\title{
Multicell Cooperation and MIMO Technologies for Broadcasting and Broadband Communications
}

\author{
Hongxiang Li, ${ }^{1}$ Lingjia Liu, ${ }^{2}$ Guoqing Li, ${ }^{3}$ Younsun Kim, ${ }^{4}$ and Jinyun Zhang ${ }^{5}$ \\ ${ }^{1}$ Department of Electrical and Computer Engineering, North Dakota State University, Fargo, ND 58108-6050, USA \\ ${ }^{2}$ Samsung Electronics, Richardson, TX 75082, USA \\ ${ }^{3}$ Intel Corporation, Hillsboro, OR 97124, USA \\ ${ }^{4}$ Samsung Electronics, Seoul, Republic of Korea \\ ${ }^{5}$ Mitsubishi Electrical Research Labs (MERL), Cambridge, MA 02139, USA
}

Correspondence should be addressed to Hongxiang Li, hongxiang.li@ndsu.edu

Received 6 April 2010; Accepted 6 April 2010

Copyright (C) 2010 Hongxiang Li et al. This is an open access article distributed under the Creative Commons Attribution License, which permits unrestricted use, distribution, and reproduction in any medium, provided the original work is properly cited.

The wireless industry is experiencing an unprecedented increase in the number and sophistication of broadcasting and broadband communication systems. The growing diffusion of new services, like mobile television and multimedia communications, emphasizes the need of advanced transmission techniques that can fundamentally increase the system capacity. In this context, the multicell collaborative transmission is becoming a major subject of research in the wireless communication community as it has been identified as one of the underlying principles for future wireless communication systems. Further, if perfect cooperation is assumed, it allows the entire network to be viewed as a single "super-MIMO" system with distributed antenna array at the base station.

This special issue aims at promoting state-of-the-art research contributions from all research areas either directly involved in or contributing to improving the issues related to multicell cooperation and MIMO technologies for broadcasting and broadband communications.

The first paper "Multiantenna analog network coding for multihop wireless networks" by R. Annavajjala et al., proposes a two-phase minimum mean-square-error bidirectional amplify and forward (MMSE-BAF) relaying protocol to allow two sources exchange independent messages via a relay node equipped with multiple antennas. The proposed protocol extends upon the so-called analog network coding schemes in the literature in that it inherently exploits the multiple antennas at the relay station to reduce the noise enhancement typical of an AF protocol and can also compensate for link imbalances between the relay and the sources and is agnostic to sources' modulation and coding schemes.

The second paper "Near optimum power control and precoding under fairness constraints in network MIMO systems" by G. Fodor et al. considers the problem of setting the uplink signal-to-noise-and-interference (SINR) target and allocating transmit powers for mobile stations in multicell spatial multiplexing wireless systems. The authors develop a numerical technique for real-time optimization of SINR targets and transmit powers. The paper also studies the impact of near optimal precoding in a multicell MIMO environment.

The third paper "Broadcast network coverage with multicell cooperation" by $\mathrm{H}$. Li et al. studies the benefits of multicell cooperation in broadcast TV network from an information theoretical perspective. The paper defines outage capacity as the figure of merit and evaluates the broadcast coverage area of multicell system where multiple base stations collaboratively transmit the broadcast signals. The results show that the coverage of a TV broadcast network can be significantly improved by multicell cooperation.

The fourth paper "An analytical multimodulus algorithm for blind demodulation in a time-varying MIMO channel context" by S. Daumont and D. Le Guennec addresses the issue of blind MIMO demodulation of communication signals, with time-varying channels and in an interception context. A new adaptive-blind source separation algorithm, which is based on the implementation of the multimodulus cost function, is proposed. 
The fifth paper "A transmit beamforming and nulling approach with distributed scheduling to improve cell edge throughput" by W. C. Wong et al. proposes a transmit scheme for WiMAX systems, where multiple base stations (BSs) employ downlink transmit beamforming and nulling for interference mitigation, with minimal coordination amongst BSs. This scheme improves system throughput and robustness, by increasing cell edge and overall cell throughputs by $68 \%$ and $19 \%$, respectively, and by delivering improvement for mobile speed up to $60 \mathrm{~km} / \mathrm{hour}$.

The sixth paper "Intercell interference coordination through limited feedback" by L. Liu et al. considers the applications of multicell transmission schemes to the downlink of future wireless communication networks. A MIMO-based scheme with limited coordination among neighboring base stations (BSs) is proposed to effectively combat the intercell interference by taking advantage of the degrees of freedom in the spatial domain.

The seventh paper "Phase-shift cyclic-delay diversity for MIMO OFDM systems" by Y. Nam et al. introduces phaseshift cyclic delay diversity (PS CDD) and space-frequencyblock-code PS CDD schemes for MIMO OFDM system. The proposed PS CDD scheme preserves the diversity advantage of traditional $\mathrm{CDD}$ in uncorrelated multiantenna channels and furthermore removes frequency-selective nulling problem of the traditional CDD in correlated multiantenna channels.

The eighth paper "Spatial domain resource sharing for overlapping cells in indoor environment" by R. Kudo et al. presents the effectiveness of spatial resource sharing among two access points. The authors introduce the primarysecondary AP scenario based on zero forcing (PSZ) and cooperative AP scenario based on zero forcing (CZ), confirming that spatial resource sharing is very attractive for the overlapping of cell scenario.

The last paper of this special issue "Robust MMSE transceiver designs for downlink MIMO systems with multicell cooperation" by J. Li et al. proposes, for joint MMSE transceiver designs, three novel robust approaches: the Robust-GIA (the most general), Robust-FIA (the most efficient), and the Robust-DCOA (which guarantees the global optimality). When cell cooperation is available, the robust approaches provide a remedy for solving the cell edge problem without reducing the number of data streams.

\section{Acknowledgments}

The authors are grateful to the reviewers for their invaluable work and to the authors of the papers collected in this special issue.

$$
\begin{array}{r}
\text { Hongxiang Li } \\
\text { Lingjia Liu } \\
\text { Guoqing Li } \\
\text { Younsun Kim } \\
\text { Jinyun Zhang }
\end{array}
$$



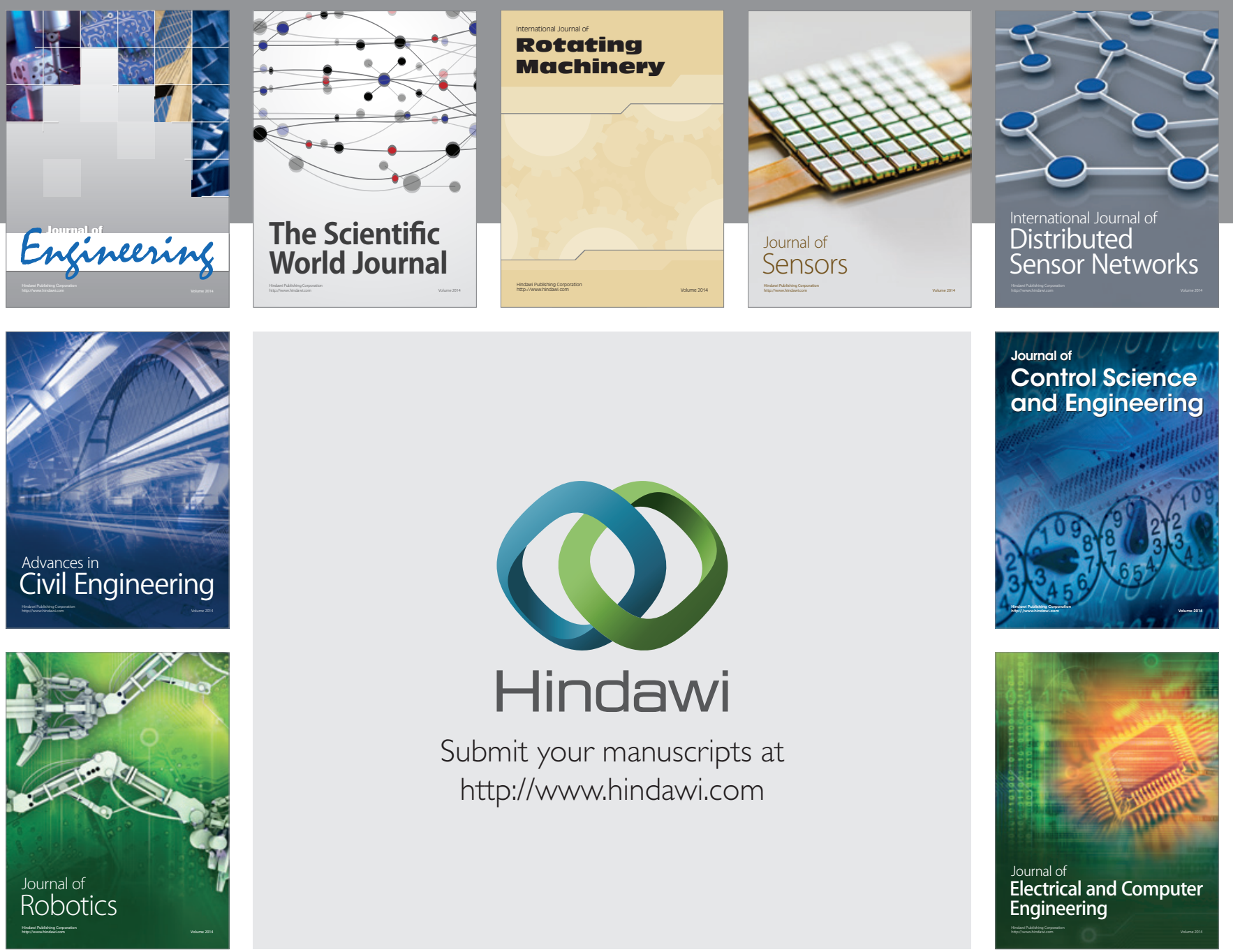

Submit your manuscripts at

http://www.hindawi.com
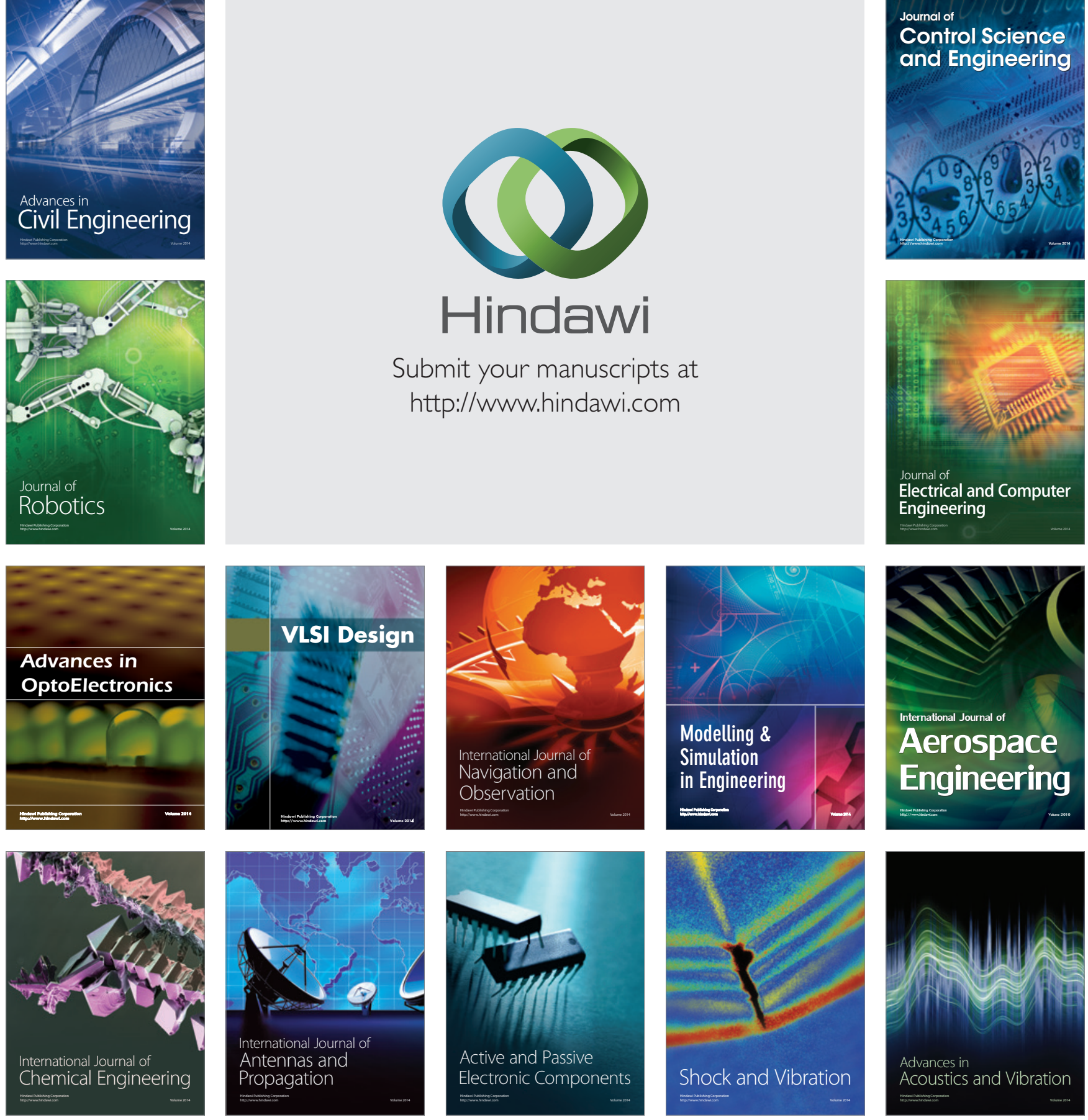